\title{
Minimalinvasive Chirurgie bei Malignomen des Gastrointestinaltrakts: Magen - Pro-Position
}

\author{
Christoph Wullstein \\ Klinik für Allgemein-, Viszeral- und Minimalinvasive Chirurgie, HELIOS Klinikum Krefeld, Deutschland
}

\section{Schlüsselwörter}

Magenkarzinom · Magenresektion · Gastrektomie · Laparoskopie · Lymphadenektomie

\section{Zusammenfassung}

Hintergrund: Wenngleich einige Studien Vorteile für laparoskopische Magenresektionen zeigen, ist die Diskussion um die laparoskopische Magenresektion beim Karzinom kontrovers. Methode: Literaturübersicht. Ergebnisse: Laparoskopische Magenresektionen beim Karzinom sind technisch sicher durchführbar und führen zu Vorteilen in der kurzfristigen Rekonvaleszenz nach der Operation im Hinblick auf geringere Schmerzen, kürzere postoperative Darmatonie, schnelleren Kostaufbau, verbesserte Lebensqualität in den ersten 3 Monaten postoperativ, eine schnellere Wiederaufnahme normaler Aktivitäten und eine kürzere Krankenhausverweildauer. Der Blutverlust ist geringer als nach offener Operation, die Operationsdauer ist länger. Einige Studien und Metaanalysen zeigen eine reduzierte Morbidität nach laparoskopischer Resektion. Die Letalität des Eingriffs unterscheidet sich nicht. Die Anzahl der bei der Operation entfernten Lymphknoten ist in vielen Studien vergleichbar, in wenigen Studien bei laparoskopischer Resektion geringer als nach offenen Operationen. Das Langzeitüberleben unterscheidet sich in den publizierten Studien nicht von dem nach konventioneller Operation. Schlussfolgerungen: Laparoskopische Magenresektionen beim Karzinom erscheinen sicher, zeigen zahlreiche Vorteile in der Rekonvaleszenz nach der Operation und sind in den Langzeitüberlebensraten vergleichbar. Möglichen Hinweisen auf eine eventuell reduzierte Anzahl an bei der Operation entnommenen Lymphknoten muss durch eine entsprechende Operationstechnik begegnet werden, die durch eine prospektive Qualitätssicherung abgesichert werden sollte.

\section{KARGER \\ Fax +497614520714 \\ Information@Karger.com}

www.karger.com (c) 2013 S. Karger GmbH, Freiburg

$1662-6664 / 13 / 0296-0356 \$ 38.00 / 0$

Accessible online at:

www.karger.com/vim
Keywords

Gastric cancer - Gastric resection - Gastrectomy .

Laparoscopy · Lymphadenectomy

\section{Summary}

Minimally Invasive Surgery for Malignancies of the Gastrointestinal Tract: Gastrectomy - Pro Position

Background: Despite several studies suggesting advantages for laparoscopic over conventional gastric resections for gastric cancer, the discussion on laparoscopic resections is still controversial. Method: Literature survey. Results: Laparoscopic gastric resections for gastric cancer are feasible and safe. Laparoscopic resections postoperatively result in an improved short-term outcome with regard to less pain, shorter duration of postoperative ileus, faster return to oral food intake, improved quality of life during 3 months postoperatively, and a shorter length of hospital stay. Blood loss associated with the operation is less during laparoscopic gastric resection than during open surgery, whereas the operation time is longer during laparoscopy. While mortality is equal, some studies show a lower morbidity following laparoscopic resections. Concerning the lymph node clearance, most studies show an equal number of lymph nodes harvested, whereas few studies have shown that laparoscopic resections resulted in a reduced number of lymph nodes harvested. The long-term survival did not differ between open and laparoscopic resections in any study. Conclusions: Laparoscopic resections for gastric cancer are safe and show several advantages in the short-term outcome. Although oncological long-term results seem to be comparable, a potential risk of a reduced number of harvested lymph nodes has to be addressed by an adequate surgical technique, which should be confirmed by prospective quality assurance. 


\section{Einleitung}

Entsprechend der interdisziplinären S3-Leitlinie «Magenkarzinom» ist die chirurgische Resektion die einzige Möglichkeit zur kurativen Behandlung und damit die Standardtherapie für alle potenziell resektablen Magenkarzinome. Standard ist die vollständige Tumorentfernung im Sinne einer R0-Resektion und die D2-Lymphadenektomie (Abb. 1), die mehr als 25 Lymphknoten beinhalten soll. Zur Frage, ob die Operation konventionell oder laparoskopisch durchgeführt werden soll/kann, wird in der Leitlinie keine Stellung bezogen [1].

Die erste laparoskopisch assistierte distale Magenresektion bei Magenkarzinom wurde 1994 von Kitano et al. [2] berichtet. Seitdem werden laparoskopische Resektionen, vor allem im asiatischen Raum, zunehmend durchgeführt. Motivation für die zunehmende Anwendung laparoskopischer Techniken sind die bei anderen Operationen gefundenen Vorteile einer kürzeren Rekonvaleszenz, einer geringeren Einschränkung der postoperativen Lungenfunktion, von weniger Schmerzen und häufig einer geringeren postoperativen Komplikationsrate $[3,4]$.

$\mathrm{Ob}$ laparoskopische Resektionen zu den konventionellen Operationstechniken onkologisch vergleichbar radikal durchgeführt werden können und zu vergleichbaren Langzeitergebnissen führen, wurde in den letzten Jahren für verschiedene Tumorentitäten hinterfragt. Am genauesten untersucht wurden diese Fragen in der chirurgischen Therapie des Kolonkarzinoms, und hier wurde zwischenzeitlich in zahlreichen prospektiv-randomisierten Studien und Metaanalysen basierend auf diesen Studien die onkologische Vergleichbarkeit auf höchstem Evidenzlevel belegt [5, 6].

Laparoskopische Resektionen beim Magenkarzinom wurden initial vor allem bei frühen Stadien eingesetzt. Viele Publikationen berichten über distale Magenresektionen bei frühen Tumorstadien. Über Resektionen bei fortgeschrittenen Tumorstadien und totale Gastrektomien mit D2-Lymphadenektomie wird weitaus seltener berichtet. Eine Schwierigkeit in der Analyse der Studien besteht in der teilweise unterschiedlichen Terminologie. Der in der englischsprachigen Literatur verwendete Begriff «gastrectomy» beinhaltet sowohl Magenteilresektionen als auch totale Gastrektomien. In asiatischen Publikationen werden häufig «distale Gastrektomien» als Operationsverfahren genannt; hierbei handelt es sich meistens um Zweidrittel-Magenteilresektionen nach europäischem Verständnis.

\section{Sicherheit laparoskopischer Magenresektionen}

Die Durchführbarkeit und Sicherheit laparoskopischer Magenresektionen beim Karzinom wurde schon recht früh in mehreren Studien gezeigt.

Die erste prospektiv-randomisierte Studie wurde 2005 von Huscher et al. [7] publiziert. In dieser Studie an 59 Patienten

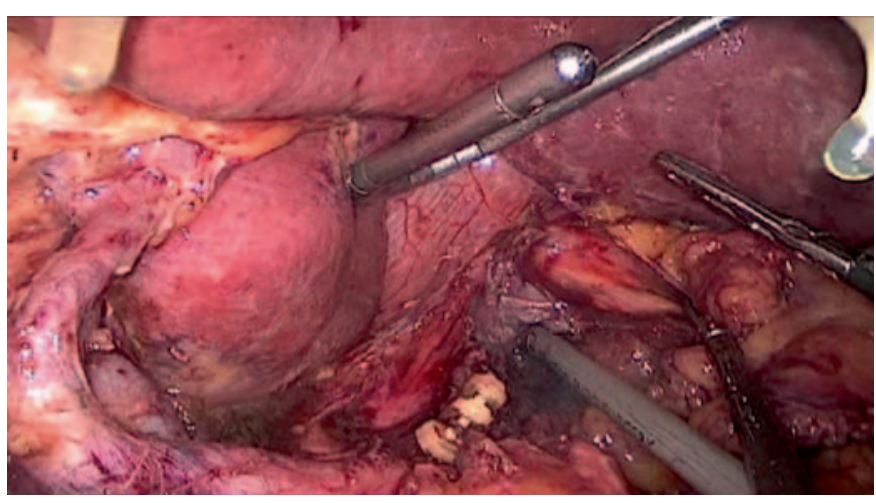

Abb. 1. Lymphadenektomie: Ausmaß bei der laparoskopischen Gastrektomie.

wurden vergleichbare Ergebnisse im Hinblick auf die Anzahl resezierter Lymphknoten (30,0 vs. 33,4), Morbidität und Letalität sowie 5-Jahres-Überlebensraten berichtet. Die Studie umfasste Patienten mit allen Tumorstadien, die durch subtotale Magenresektion behandelt wurden.

Kitano et al. [8] berichteten 2007 über die Ergebnisse von 1294 laparoskopischen Magenresektionen, die in 16 japanischen Zentren durchgeführt wurden. 93,7\% der Patienten dieser Studie wiesen ein Union for International Cancer Control(UICC)-Stadium 1 auf. Die weitaus häufigste Operation in dieser Studie war die distale Magenresektion mit 91,5\%; Gastrektomien wurden bei 4,3\% der Patienten durchgeführt. In dieser ersten großen Studie betrug die intraoperative Komplikationsrate $1,9 \%$, die Konversionsrate 1,1\%. Postoperative Komplikationen traten bei $12,9 \%$ der Patienten auf. Die 5-Jahres-Überlebensraten in dieser Studie betrugen 99,8\% für Stadium 1a und 98,7\% für Stadium 1b. Diese große Studie zeigte die operative und onkologische Sicherheit der laparoskopischen Resektion bei frühen Stadien des Magenkarzinoms.

Eine weitere frühe, retrospektiv vergleichende Studie zeigte die Sicherheit laparoskopischer subtotaler Magenresektionen und Gastrektomien im Vergleich zu konventionellen Techniken [9].

Im Jahr 2009 publizierten Jeong et al. [10] eine Subgruppenanalyse von 131 Patienten mit laparoskopischen Gastrektomien aus einer retrospektiven koreanischen Studie an 1485 Patienten mit laparoskopischen Magenresektionen. Die Anzahl entfernter Lymphknoten betrug in dieser Studie 34,7, die postoperative Morbidität lag bei 19\%, die Konversionsrate war $0,8 \%$.

\section{Kurzfristige Rekonvaleszenz}

Im Hinblick auf die kurzfristige Rekonvaleszenz nach Magenresektionen zeigen die meisten Studien Vorteile für das laparoskopische Vorgehen [11, 12].

Strong et al. [13] zeigten in einer Case-Match-Studie zur subtotalen Magenresektion Vorteile der Laparoskopie im 
Hinblick auf den postoperativen Schmerzmittelbedarf, auf die Länge der Krankenhausaufenthaltsdauer und auf die Spätkomplikationen. Die Operationszeit war bei laparoskopischen Operationen länger als bei offenen Operationen.

Eine prospektiv-randomisierte Studie mit 164 Patienten aus Korea zeigt neben Vorteilen bezüglich des intraoperativen Blutverlusts, postoperativer Schmerzen und der Krankenhausverweildauer auch Vorteile in der Lebensqualität bis 3 Monate postoperativ nach laparoskopischer distaler Magenresektion bei Magenfrühkarzinomen [14].

Eine aktuelle prospektiv-randomisierte Studie, die für die Patienten verblindet durchgeführt wurde, zeigt ebenfalls weniger Schmerzen, eine kürzere Krankenhausverweildauer und schnellere Wiederherstellung der physischen Aktivität nach laparoskopischer im Vergleich zur offenen distalen Magenresektion [15].

Eine frühe Metaanalyse von Yakoub et al. [16] aus dem Jahr 2009 fasst die Ergebnisse von neun retrospektiven Studien und drei kontrolliert randomisierten Studien zur distalen Magenresektion zusammen. In dieser Analyse zeigt sich eine durch die laparoskopische Operation um 54 min verlängerte Operationsdauer, ein um $151 \mathrm{ml}$ reduzierter intraoperativer Blutverlust, eine um einen Tag frühere orale Nahrungsaufnahme, ein um einen Tag zügigeres Wiedereinsetzen der Darmtätigkeit, ein um 2 Tage verkürzter Schmerzmittelbedarf und eine verkürzte Zeit bis zur Mobilisierung der Patienten. Die Morbidität war nach laparoskopischer Resektion mit 10,5\% geringer als nach offener Resektion mit 20,1\% ( $\mathrm{p}=0,003)$.

Eine andere Metaanalyse aus dem Jahr 2011 umfasst die Ergebnisse von fünf prospektiv-randomisierten Studien [17]. Insgesamt wurden die Ergebnisse von 326 Patienten mit distalen Magenresektionen ausgewertet. Auch diese Analyse zeigt Vorteile im Hinblick auf den intraoperativen Blutverlust, eine kürzere Krankenhausverweildauer und einen geringeren postoperativen Schmerzmittelbedarf. Diese Metaanalyse zeigt darüber hinaus eine geringere Komplikationsrate nach laparoskopischer als nach konventioneller Magenresektion (Odds Ratio (OR): 0,38; 95\% Konfidenzintervall (CI): 0,19-0,77; $\mathrm{p}=$ 0,007). Es bestand ein Trend zu einer höheren pulmonalen Komplikationsrate nach offener Magenresektion $(\mathrm{p}=0,06)$. Im Hinblick auf die Letalität zeigte sich kein Unterschied.

Bezüglich der totalen Gastrektomie mit D2-Lymphadenektomie liegt eine größere retrospektive Studie vor. Shinohara et al. [18] verglichen in einer Matched-Pair-Analyse die Ergebnisse von 186 laparoskopisch mit 150 offen gastrektomierten Patienten. In dieser Studie zeigen sich Vorteile für die Laparoskopie den intraoperativen Blutverlust (mit einer um zwei Drittel reduzierten Bluttransfusionsrate) und die Krankenhausverweildauer betreffend. Auch hier war die Operationszeit der laparoskopischen Operation verlängert. Bezüglich der Morbidität und Letalität bestanden keine Unterschiede zwischen den Verfahren.

Eine weitere Studie befasst sich mit totalen Gastrektomien: Jeong et al. [19] untersuchten in einer Matched-Pair-
Analyse 122 laparoskopische und 122 offene Gastrektomien. Während in dieser Studie vergleichbare Ergebnisse für die offene und laparoskopische Technik beschrieben wurden, zeigt diese Studie im Gegensatz zu allen anderen Studien in einer Subgruppenanalyse eine erhöhte Morbidität für die laparoskopische Gastrektomie mit D2-Lymphadenektomie.

Verschiedene Studien zeigen, dass die postoperative Komplikationsrate bei laparoskopischen und offenen Magenresektionen vergleichbar ist [7, 20-22]. Neben den Metaanalysen aus den Jahren 2009 und 2011 [16, 17] zeigt eine aktuelle Metaanalyse aus 2013 (15 Studien, 2519 Patienten) ebenfalls, dass die postoperative Morbidität nach laparoskopischer Magenresektion, auch bei fortgeschrittenen Tumorstadien, geringer ist als nach offenen Operationen, sowohl die Gesamtkomplikationsrate als auch die chirurgischen Komplikationen betreffend [23].

\section{Onkologische Ergebnisse}

Die Frage, ob bei laparoskopischen Magenresektionen eine adäquate Lymphadenektomie möglich ist, wird in verschiedenen Studien adressiert (Tab. 1). Während die meisten Single-Center-Studien eine vergleichbare Anzahl an entfernten Lymphknoten vorweisen, zeigen von den vier Metaanalysen drei eine geringere Lymphknotenanzahl nach laparoskopischer Resektion.

In den Metaanalysen von Yakoub et al. [16] und Ohtani et al. [17] werden laparoskopisch jeweils etwa 5 Lymphknoten weniger entfernt als bei konventionellen Magenresektionen. Die Metaanalyse von Zeng et al. [24] von 2012 zeigt in der Gesamtanalyse keinen Unterschied in der Lymphadenektomie. Wurden in der Analyse jedoch die Studien, die zu einer hohen Heterogenität der Analyse beitrugen, ausgeschlossen, zeigte sich, dass in der laparoskopischen Gruppe etwas weniger Lymphknoten entfernt wurden. Die aktuellste Metaanalyse von Chen et al. [23] aus 2013 zeigt keinen Unterschied in der Anzahl entfernter Lymphknoten.

Interessant ist die Studie von Kim et al. [25] aus dem Jahr 2005. Diese Studie zeigt die Anzahl der entfernten Lymphknoten aufgeschlüsselt nach der Art der angestrebten Lymphadenektomie. In dieser Studie zeigten sich für D1 $+\alpha$ und D1 $+\beta$ keine Unterschiede zwischen der offenen und der laparoskopischen Lymphadenektomie. Für die D2-Lymphadenektomie bestand in dieser Studie ein Unterschied zugunsten der konventionell operierten Patienten (Tab. 1).

\section{Onkologische Langzeitergebnisse}

Wenngleich das Ausmaß der erreichten Radikalität die Lymphadenektomie betreffend nicht einheitlich $\mathrm{zu}$ sein scheint, zeigen doch alle Studien, die vergleichend Tumorrezidivraten und Langzeitüberlebensraten angeben, übereinstimmend keine Unterschiede zwischen konventionellen und laparoskopischen Resektionen [7, 11, 18, 26-30]. 
Tab. 1. Bei der Lymphadenektomie entfernte Lymphknoten: Vergleich laparoskopische und konventionelle Resektion

\begin{tabular}{|c|c|c|c|c|c|c|c|}
\hline \multirow[t]{2}{*}{ Autoren } & \multirow[t]{2}{*}{ Operation } & \multirow{2}{*}{$\begin{array}{l}\text { Ausmaß der } \\
\text { Lymphadenektomie }\end{array}$} & \multirow[t]{2}{*}{ Art der Studie } & \multirow[t]{2}{*}{ N (MIC/offen) } & \multicolumn{2}{|c|}{ Anzahl entfernter Lymphknoten } & \multirow[t]{2}{*}{$\mathrm{p}$} \\
\hline & & & & & MIC & offen & \\
\hline $\begin{array}{c}\text { Kawamura et al., } \\
2008 \text { [38] }\end{array}$ & $\begin{array}{l}\text { distale } \\
\text { Resektion }\end{array}$ & $\mathrm{D} 2$ & retro & $120(53 / 67)$ & 49,2 & 44,8 & n.s. \\
\hline $\begin{array}{l}\text { Huscher et al., } \\
\quad 2005 \text { [7] }\end{array}$ & $\begin{array}{l}\text { subtotale } \\
\text { Resektion }\end{array}$ & D2 & RCT & $59(30 / 29)$ & 30 & 33,4 & n.s. \\
\hline \multirow{4}{*}{$\begin{array}{l}\text { Kim et al., } \\
2005 \text { [25] }\end{array}$} & \multirow{4}{*}{$\begin{array}{l}\text { subtotale } \\
\text { Resektion }\end{array}$} & gesamt & \multirow[t]{4}{*}{ retro } & $147(71 / 76)$ & 22,8 & 27,4 & 0,0123 \\
\hline & & $\mathrm{D} 1+\mathrm{Nr} .7$ & & $(11 / 3)$ & 16,5 & 9,0 & 0,1617 \\
\hline & & $\mathrm{D} 1+$ Nr. $7,8 \mathrm{a}, 9$ & & $(42 / 34)$ & 23,7 & 21,6 & 0,3785 \\
\hline & & D2 & & $(18 / 39)$ & 24,5 & 33,9 & 0,0019 \\
\hline $\begin{array}{l}\text { Strong et al., } \\
2009 \text { [13] }\end{array}$ & $\begin{array}{l}\text { subtotale } \\
\text { Resektion }\end{array}$ & D2 & $\begin{array}{l}\text { Case-Match } \\
\text { retro }\end{array}$ & $(30 / 30)$ & 18 & 21 & 0,03 \\
\hline $\begin{array}{l}\text { Hamabe et al., } \\
\quad 2012 \text { [39] }\end{array}$ & distal, total & D2 & retro & $(66 / 101)$ & 63,7 & 44 & $<0,0001$ \\
\hline $\begin{array}{c}\text { Shinohara et al., } \\
2013 \text { [18] }\end{array}$ & $\begin{array}{l}\text { distal, subtotal, } \\
\text { total }\end{array}$ & $\mathrm{D} 2$ & $\begin{array}{l}\text { Case-Match } \\
\text { retro }\end{array}$ & $(186 / 123)$ & 45,3 & 43,8 & 0,446 \\
\hline \multirow{2}{*}{$\begin{array}{l}\text { Sato et al., } \\
\quad 2012 \text { [21] }\end{array}$} & \multirow[t]{2}{*}{ verschiedene } & $\mathrm{D} 1, \mathrm{D} 1+$ & \multirow[t]{2}{*}{ retro } & $(122 / 44)$ & 26 & 21 & $<0,01$ \\
\hline & & $\mathrm{D} 2$ & & $(36 / 130)$ & 32 & 35 & n.s. \\
\hline
\end{tabular}

Die 3- und 5-Jahres-Überlebensraten von zwölf Studien werden in der aktuellen Metaanalyse von Chen et al. [23] aufgeführt, und es findet sich ebenfalls kein Unterschied zwischen beiden Verfahren.

\section{Lernkurve}

Bei vielen laparoskopischen Eingriffen wird die sogenannte Lernkurve hinterfragt. Eine Diskussion über Lernkurven bei offenen, konventionellen Operationen unterbleibt indes regelmäßig.

Magenresektionen sind offen wie laparoskopisch komplexe Eingriffe, deren Schwierigkeitsgrad mit dem Ausmaß der Lymphadenektomie weiter zunimmt. Bezüglich der Lernkurven bei laparoskopischen Magenresektionen gibt es eine erhebliche Schwankungsbreite, die in der Metaanalyse von Zeng et al. [24] basierend auf den unterschiedlichen eingeschlossenen Studien zwischen 20 und 300 variiert, mit einer Häufung der Angaben um die 30 Operationen.

Kim et al. [31] zeigen in ihrer Studie, dass die Lernkurve für die distale Magenresektion nach 20-40 Operationen abgeschlossen ist.

Mit der Frage, wie sich die Lernkurve bei Weiterbildungsassistenten in einem bereits etablierten Setting verhält, befassen sich die Studien von Nunobe et al. [32] und Tokunaga et al. [33]. Nunobe et al. [32] beschreiben einen strukturierten Ausbildungsalgorithmus. Trainiert wurden Assistenten mit 7-13 Jahren chirurgischer Erfahrung, die jeweils über Erfahrungen mit 20-50 konventionellen Gastrektomien und 10-30 laparoskopischen Cholezystektomien verfügten. Diese Assistenten wurden dann für jeweils 30-35 laparoskopische Ma- genresektionen zunächst als Kameraassistent, dann als erster Operationsassistent eingesetzt. Nach dieser Vorbereitung war die individuelle Lernkurve als Operateur nach sechs eigenständig durchgeführten Operationen abgeschlossen und erreichte das Plateau des ausbildenden Chirurgen.

Tokunaga et al. [33] zeigten, dass in einem etablierten Programm nach je 20 Kameraassistenzen und 20 ersten Operationsassistenzen keine messbare Lernkurve im Hinblick auf Blutungen, Komplikationen oder Anzahl entfernter Lymphknoten zu verzeichnen war. Bezüglich der Operationszeit bestand ein gewisser Lerneffekt [33].

\section{Diskussion}

Die Vorteile von laparoskopischen Operationen für die Patienten sind für viele Indikationen gut belegt. Die Patienten profitieren von geringeren Schmerzen und einer schnelleren Erholung. Die postoperative Einschränkung der Lungenfunktion ist nach laparoskopischen Eingriffen weniger ausgeprägt als bei offenen Operationen, was sich in zum Teil reduzierten pulmonalen Komplikationen widerspiegelt. Während laparoskopische Operationen für viele Indikationen unumstritten und z.B. Resektionen beim Kolonkarzinom mit höchster Evidenz akzeptiert sind [34], werden laparoskopische Resektionen am oberen Gastrointestinaltrakt besonders bei onkologischen Erkrankungen weiterhin kontrovers diskutiert.

Die Evidenz für eine Bewertung laparoskopischer Magenresektionen nimmt langsam zu, wobei sich viele Studien mit distalen und subtotalen Magenresektionen befassen und auch das Ausmaß der analysierten Lymphadenektomie variiert. 
Als sicher belegt können inzwischen die bekannten Vorteile laparoskopischer Techniken auch für Magenresektionen gelten. Mit einem Evidenzlevel von 1-2 führen laparoskopische Magenresektionen zu einem niedrigeren Blutverlust, zu geringeren postoperativen Schmerzen und zu einer kürzeren Krankenhausverweildauer. Einige Studien zeigen darüber hinaus ein schnelleres Wiedereinsetzen der Darmfunktion und eine schnellere Normalisierung der körperlichen Belastbarkeit. Eine Verbesserung der postoperativen Lebensqualität zeigt eine Studie [14].

Das Erreichen eines geringeren intraoperativen Blutverlustes, wie in allen Studien gezeigt wird, und einer geringeren Transfusionsrate, wie die Arbeit von Shinohara et al. [18] zeigt, kann durchaus von onkologischer Bedeutung sein.

So besteht eine umfassende Evidenz, dass die perioperative Gabe von Bluttransfusionen beim kolorektalen Karzinom ein unabhängiger Risikofaktor für das Entstehen von Tumorrezidiven ist [35]. Auch für das Magenkarzinom wurde in einer aktuellen Arbeit aus dem Jahr 2013 gezeigt, dass die Höhe des operativen Blutverlustes ein unabhängiger Risikofaktor für das tumorbezogene Langzeitüberleben nach kurativer Resektion von Magenkarzinomen ist [36].

Mehrfach wurde eine Reduktion der postoperativen Morbidität durch die laparoskopische Operation gezeigt. Dieser Effekt scheint bei distalen Magenresektionen ausgeprägter zu sein als bei totalen Gastrektomien. Für die Gastrektomie wird überwiegend eine der offenen Operation vergleichbare Morbidität beschrieben. Eine niedrige Morbidität erscheint auch im Hinblick auf die onkologischen Langzeitergebnisse erstrebenswert. Li et al. [37] haben 2013 eine Studie publiziert, in der die postoperative Morbidität ein unabhängiger Prognoseparameter für das 5-Jahres-Überleben nach radikaler Magenoperation bei Karzinom ist.

Die Bewertung der onkologischen Wertigkeit der laparoskopischen Operation selbst bei Magenkarzinom ist nicht ein- heitlich, und eine abschließende Beurteilung der Anzahl der entfernten Lymphknoten ist schwierig. Die bisher vorliegenden Metaanalysen zeigen überwiegend, dass die Laparoskopie zu einer verminderten Anzahl an entfernten Lymphknoten führen kann. Vor allem aktuelle Studien und die aktuellste und umfassendste Metaanalyse [23] scheinen hier zwar zunehmend eine Gleichwertigkeit zu zeigen, dennoch muss dieser Punkt als kritisch angesehen werden. Allerdings zeigen bisher alle Studien mit Langzeitergebnissen keine das Langzeitüberleben nach 3 und 5 Jahren betreffenden Unterschiede zwischen offenen und laparoskopischen Operationen, sodass eine eventuell reduzierte Zahl an entnommenen und untersuchten Lymphknoten sich bisher nicht in schlechteren Überlebensraten wiederfindet.

\section{Fazit}

Laparoskopische Magenresektionen bei Karzinom erscheinen nach Studienlage sicher und gehen, wie andere laparoskopische Operationen auch, mit zahlreichen Vorteilen in der kurzfristigen Rekonvaleszenz nach der Operation selbst einher. Den Hinweisen auf eine möglicherweise geringere Lymphknotenausbeute muss durch eine sorgfältige, der konventionellen Technik genau entsprechende Lymphadenektomie begegnet werden. Diese sollte durch eine prospektive Datenerhebung qualitätskontrolliert werden. Entsprechend der S3-Leitlinie sollen für eine D2-Lymphadenektomie mehr als 25 Lymphknoten entfernt und untersucht werden.

\section{Disclosure Statement}

Es bestehen keine Interessenkonflikte.

\section{Literatur}

1 Moehler M, Al-Batran SE, Andus T, et al: S3-Leitlinie «Magenkarzinom»- Diagnostik und Therapie der Adenokarzinome des Magens und oesophagogastralen Übergangs. Z Gastroenterol 2011;49:461531.

2 Kitano S, Iso Y, Moriyama M, Sugimachi K: Laparoscopy-assisted Billroth I gastrectomy. Surg Laparosc Endosc 1994:4:146-148.

3 Abraham NS, Young JM, Solomon MJ: Meta-analysis of short-term outcomes after laparoscopic resection for colorectal cancer. Br J Surg 2004;91: 1111-1124

4 Schwenk W, Haase O, Neudecker J, Müller JM Short term benefits for laparoscopic colorectal resection. Cochrane Database Syst Rev 2005;(3): CD003145.

5 Bonjer HJ, Hop WC, Nelson H, et al: Laparoscopically assisted vs open colectomy for colon cancer: a meta-analysis. Arch Surg 2007;142:298-303.
6 Kuhry E, Schwenk W, Gaupset R, Romild U, Bonjer J: Long-term outcome of laparoscopic surgery for colorectal cancer: a Cochrane systematic review of randomised controlled trials. Cancer Treat Rev 2008;34:498-504.

7 Huscher CGS, Mingoli A, Sgarzini G, Sansonetti A, Di Paola M, Recher A, Ponzano C: Laparoscopic versus open subtotal gastrectomy for distal gastric cancer - five-year results of a randomized prospective trial. Ann Surg 2005;241:232-237.

8 Kitano S, Shiraishi N, Uyama I, Sugihara K, Tanigawa N; the Japanese Laparoscopic Surgery Study Group: A multicenter study on oncologic outcome of laparoscopic gastrectomy for early cancer in Japan. Ann Surg 2007;245:68-72.

9 Pugliese R, Maggioni D, Sansonna F, Scandroglio I, Ferrari GC, Di Lernia S, Costanzi A, Pauna J, de Martini P: Total and subtotal laparoscopic gastrectomy for adenocarcinoma. Surg Endosc 2007;21: 21-27.
10 Jeong G-A, Cho G-S, Kim H-H, Lee H-J, Ryu S-W, Song K-Y: Laparoscopy-assisted total gastrectomy for gastric cancer: a multicenter retrospective analysis. Surgery 2009;146:469-474.

11 Koeda K, Nishizuka S, Wakabayashi G: Minimally invasive surgery for gastric cancer: the future standard of care. World J Surg 2011;35:1469-1477.

12 Kunisaki C, Makino H, Kosaka T, Oshima T, Fujii S, Takagawa R, Kimura J, Ono HA, Akiyama H, Taguri M, Morita S, Endo I: Surgical outcomes of laparoscopy-assisted gastrectomy versus open gastrectomy for gastric cancer: a case-control study. Surg Endosc 2012;26:804-810.

13 Strong VE, Devaud N, Allen PJ, Gonen M, Brennan MF, Coit D: Laparoscopic versus open subtotal gastrectomy for adenocarcinoma: a case-control study. Ann Surg Oncol 2009;16:1507-1513. 
14 Kim Y-W, Baik YH, Yun YH, Nam BH, Kim DH, Choi IJ, Bae J-M: Improved quality of life outcomes after laparoscopy-assisted distal gastrectomy for early gastric cancer - results of a prospective randomized clinical trial. Ann Surg 2008;248:721-727.

15 Takiguchi S, Fujiwara Y, Yamasaki M, Miyata H, Nakajima K, Sekimoto M, Mori M, Doki Y: Laparoscopy-assisted distal gastrectomy versus open distal gastrectomy: a prospective randomized single-blind study. World J Surg 2013;37:2379-2386.

16 Yakoub D, Athanasiou T, Tekkis P, Hanna GB: Laparoscopic assisted distal gastrectomy for early gastric cancer: is it an alternative to the open approach? Surg Oncol 2009;18:322-333.

17 Ohtani H, Tamamori Y, Noguchi K, Azuma T, Fujimoto S, Oba H, Aoki T, Minami M, Hirakawa K Meta-analysis of laparoscopy-assisted and open distal gastrectomy for gastric cancer. J Surg Res 2011:171:479-485.

18 Shinohara T, Satoh S, Kanaya S, Ishida Y, Taniguchi K, Isogaki J, Inaba K, Yanaga K, Uyama I: Laparoscopic versus open D2 gastrectomy for advanced gastric cancer: a retrospective cohort study. Surg Endosc 2013;27:86-94.

19 Jeong O, Jung MR, Kim GY, Kim HS, Ryu SY, Park YK: Comparison of short-term surgical outcomes between laparoscopic and open total gastrectomy for gastric carcinoma: case-control study using propensity score matching method. J Am Coll Surg 2013;216:184-191.

20 Kim H-H, Hyung WJ, Cho GS, Kim MC, Han S-U, Kim W, Ryu S-W, Lee H-J, Song KY: Morbidity and mortality of laparoscopic gastrectomy versus open gastrectomy for gastric cancer, an interim report - a phase III multicenter, prospective, randomized trial (KLASS trial). Ann Surg 2010;251:417-420.

21 Sato H, Shimada M, Kurita N, Iwata T, Nishioka M, Morimoto S, Yoshikawa K, Miyatani T, Goto M, Kashihara H, Takasu C: Comparison of longterm prognosis of laparoscopy-assisted gastrectomy and conventional open gastrectomy with special reference to D2 lymph node dissection. Surg Endosc 2012;26:2240-2246.

22 Tokunaga M, Kondo J, Tanizawa Y, Bando E, Kawamura $\mathrm{T}$, Terashima M: Postoperative intraabdominal complications assessed by the ClavienDindo classification following open and laparoscopy-assisted distal gastrectomy for early gastric cancer. J Gastrointest Surg 2012;16:1854-1859.
3 Chen K, Xu X-W, Mou Y-P, Pan Y, Zhou Y-C, Zhang R-C, Wu D: Systematic review and metaanalysis of laparoscopic and open gastrectomy for advanced gastric cancer. World J Surg Oncol 2013; 11:182.

24 Zeng Y-K, Yang Z-L, Peng J-S, Lin H-S, Cai L: Laparoscopy-assisted versus open distal gastrectomy for early gastric cancer - evidence from randomized and nonrandomized clinical trials. Ann Surg 2012;256:39-52.

25 Kim M-C, Kim K-H, Kim H-H, Jung G-J: Comparison of laparoscopy-assisted by conventional open distal gastrectomy and extraperigastric lymph node dissection in early gastric cancer. J Surg Oncol 2005;91:90-94.

26 Kim K-H, Kim M-C, Jung G-J, Choi H-J, Jang J-S, Kwon H-C: Comparative analysis of five-year survival results of laparoscopy-assisted gastrectomy versus open gastrectomy for advanced gastric cancer: a case-control study using a propensity score method. Dig Surg 2012;29:165-171.

27 Moisan F, Norero E, Slako M, Varas J, Palominos G, Crovari F, Ibanez L, Perez G, Pimentel F, Guzmán S, Jarufe N, Boza C, Escalona A, Funke R: Completely laparoscopic versus open gastrectomy for early and advanced gastric cancer: a matched cohort study. Surg Endosc 2012;26:661-672.

28 Park DJ, Han S-U, Hyung WJ, Kim MC, Kim W, Ryu SY, Ryu S-W, Song KY, Lee H-J, Cho G-S, Kim H-H; Korean Laparoscopic Gastrointestinal Surgery Study (KLASS) Group: Long-term outcomes after laparoscopy-assisted gastrectomy for advanced gastric cancer: a large-scale multicenter retrospective study. Surg Endosc 2012;26:1548-1553.

29 Song J, Lee H-J, Cho GS, Han S-U, Kim M-C, Ryu SW, Kim W, Song KY, Kim H-H, Hyung WJ; Korean Laparoscopic Gastrointestinal Surgery Study (KLASS) Group: Recurrence following laparoscopy-assisted gastrectomy for gastric cancer: a multicenter retrospective analysis of 1,417 patients. Ann Surg Oncol 2010;17:1777-1786.

30 Hwang S-H, Park DJ, Jee YS, Kim M-C, Kim H-H, Lee H-J, Yang H-K, Lee KU: Actual 3-year survival after laparoscopy-assisted gastrectomy for gastric cancer. Arch Surg 2009;144:559-564.
1 Kim H-G, Park J-H, Jeong S-H, Lee Y-J, Ha W-S, Choi S-K, Hong S-C, Jung E-J, Ju J-T, Jeong C-Y, Park T: Totally laparoscopic distal gastrectomy after learning curve completion: comparison with laparoscopy-assisted distal gastrectomy. J Gastric Cancer 2013;13:26-33.

32 Nunobe S, Hiki N, Tanimura S, Nohara K, Sano T, Yamaguchi T: The clinical safety of performing laparoscopic gastrectomy for gastric cancer by trainees after sufficient experience in assisting. World J Surg 2013;37:424-429.

33 Tokunaga M, Hiki N, Fukunaga T, Miki A, Ohyama S, Miyata S, Yamaguchi T: Learning curve of laparoscopy-assisted gastrectomy using a standardized surgical technique and an established educational system. Scand J Surg 2011;100:86-91.

34 Leitlinienprogramm Onkologie (Deutsche Krebsgesellschaft, Deutsche Krebshilfe, AWMF): S3Leitlinie Kolorektales Karzinom. Langversion 1.0, AWMF Registrierungsnummer 021-007.OL. http:// leitlinienprogramm-onkologie.de/Leitlinien.7.0.html.

35 Amato A, Pescatori M: Perioperative blood transfusions for the recurrence of colorectal cancer. Cochrane Database Syst Rev 2006;(1):CD005033.

36 Liang Y-X, Guo H-H, Deng J-Y, Wang B-G, Ding $\mathrm{X}-\mathrm{W}$, Wang $\mathrm{X}-\mathrm{N}$, Zhang L, Liang H: Impact of intraoperative blood loss on survival after curative resection for gastric cancer. World J Gastroenterol 2013;19:5542-5550.

37 Li Q-G, Li P, Tang D, Chen J, Wang D-R: Impact of postoperative complications on long-term survival after radical resection for gastric cancer. World J Gastroenterol 2013;19:4060-4065.

38 Kawamura H, Homma S, Yokota R, Yokota K, Watarai H, Hagiwara M, Sato M, Noguchi K, Ueki S, Kondo Y: Inspection of safety and accuracy of D2 lymph node dissection in laparoscopy-assisted dista gastrectomy. World J Surg 2008;32:2366-2370.

39 Hamabe A, Omori T, Tanaka K, Nishida T: Comparison of long-term results between laparoscopyassisted gastrectomy and open gastrectomy with D2 lymph node dissection for advanced gastric cancer. Surg Endosc 2012;26:1702-1709. 